\title{
Diagnostic Coding for Intramucosal Carcinoma and Neuroendocrine Tumor in the Colorectum: Proposal for Avoiding Confusing Coding in Korea
}

\author{
Dong Soo Han ${ }^{1, *}$, Jin Hee Sohnn ${ }^{2, *}$, Jeong-Sik Byeon ${ }^{3}$, Hwang Choi ${ }^{4}$ and Joon Mee Kim ${ }^{5}$ \\ ${ }^{1}$ Department of Internal Medicine, Hanyang University Guri Hospital, Hanyang University College of Medicine, Guri, ${ }^{2}$ Department of Pathology, \\ Kangbuk Samsung Hospital, Sungkyunkwan University School of Medicine, Seoul, ${ }^{3}$ Department of Gastroenterology, Asan Medical Center, \\ University of Ulsan College of Medicine, Seoul, ${ }^{4}$ Department of Internal Medicine, Incheon St. Mary's Hospital, College of Medicine, The Catholic \\ University of Korea, Incheon, ${ }^{5}$ Department of Pathology, Inha University Hospital, Inha University School of Medicine, Incheon, Korea
}

\begin{abstract}
Applying proper coding is important for doctors practicing gastroenterology. The coding systems established by various organizations define tumors differently. As a result of changing concepts of tumor classification, there are coding and reimbursement issues following the confirmation of malignant lesions by nationwide cancer screening in patients with intramucosal carcinoma and neuroendocrine tumors of the colorectum. In addition, there have been discrepancies between the views of endoscopists and pathologists regarding tumor coding. The Korean Society of Gastrointestinal Endoscopy held an expert meeting and established a consensus for the coding of intramucosal carcinoma and neuroendocrine tumor of the colorectum.
\end{abstract}

Key Words: Intramucosal carcinoma; Neuroendocrine tumors; Clinical coding

\section{INTRODUCTION}

The frequency of endoscopic polypectomy during screening colonoscopy has increased and has become more convenient due to the National Colon Cancer Screening Program and easier bowel preparation methods. In addition, advances in endoscopic techniques are allowing endoscopic removal of sessile polyps and early colorectal tumors. However, with increasing financial support from the government for the financial expense of cancer treatment and increasing rates of purchase of private cancer insurance, not only medical but also social concerns have been augmented. These public and private forms of coverage for medical expense reimbursement differ with the given disease code, but vague definition and

Received: October 16, 2014 Revised: December 8, 2014

Accepted: December 9, 2014

Correspondence: Dong Soo Han

Department of Internal Medicine, Hanyang University Guri Hospital, Hanyang University College of Medicine, 153 Gyeongchun-ro, Guri 471-701, Korea

Tel: +82-31-560-2226, Fax: +82-31-555-2998, E-mail: hands@hanyang.ac.kr

*Dong Soo Han and Jin Hee Sohn contributed equally to this work as first authors.

(c) This is an Open Access article distributed under the terms of the Creative Commons Attribution Non-Commercial License (http://creativecommons.org/ licenses/by-nc/3.0) which permits unrestricted non-commercial use, distribution, and reproduction in any medium, provided the original work is properly cited. classification of cancer are leading to confusion in society.

There are various diagnostic criteria and classification and staging systems, and the results for colorectal cancer (CRC) can alter according to the system that is applied. ${ }^{2}$ Significant time and effort are required to become familiar with changing criteria or standards for the diagnosis of CRC.

Considerable numbers of endoscopists and pathologists are familiar with the Japanese Classification of Colorectal Carcinoma. ${ }^{3}$ On the other hand, the updated Vienna classification was not as accepted by both groups. There was a discrepancy in the criteria for financial reimbursement between the government and private insurance companies. To solve this problem, the Gastrointestinal Pathology Study Group of the Korean Society of Pathologists has made continuous and steady efforts to minimize the discrepancies among pathologists in the diagnostic criteria for CRC. 4,5

However, many institutes still use different guidelines in diagnosing and classifying malignancy, which may result in confusion in the clinical setting. In addition, many problems may arise from endoscopically acquired specimens, due to a lack of consensus between pathologists and endoscopists.

To resolve these conflicts, the Korean Society of Gastrointestinal Endoscopy invited specialists from the fields of gastro- 
enterology, pathology, law, and others to exchange opinions through discussion, with the goals of coming to understand and then improve the current status concerning different coding issues. This article is written to analyze and verify the aims based on the results obtained.

\section{CODING FOR EPITHELIAL NEOPLASM IN COLON}

The International Classification of Diseases (ICD) and related health problems established by the World Health Organization (WHO) is the standard diagnostic tool for epidemiology, health management, and clinical purposes. It includes the analysis of the general health situation of population groups and is used to monitor the incidence and prevalence of diseases and other health problems in the clinical setting. ${ }^{6}$

The Korean Standard Classification of Diseases (KCD) was established with the translation of the ICD by Statistics Korea to allow communication in a common language in clinical settings. Malignant neoplasms are assigned a " $\mathrm{C}$ code" and are classified as C00 to C97 according to their anatomical location. All forms of malignancy that are possible in a particular anatomical location are included, such as carcinoma including squamous cell carcinoma and adenocarcinoma, sarcoma, soft tissue malignancy including mesothelioma, lymphoma, leukemia, and even unidentified cancer.

Malignant neoplasm of the colon is classified as C18 (colon), C19 (rectosigmoid junction), or C20 (rectum) according to the location of the malignancy. Carcinoma in situ is defined to be a conformational change that is between dysplasia and invasive cancer. It is classified as D00 to D09 according to its anatomical location, and D00 and D01 are assigned to carcinoma in situ of the gastrointestinal tract. This is then subclassified as D01.0 (colon), D01.1 (rectosigmoid junction), or D01.2 (rectum). Colon adenoma is a benign neoplasm (D10 to D36) coded as D12. Neoplasm of uncertain or unknown behavior of the digestive system is classified as D37. Pathologically, if the malignant cells do not infiltrate through the basement membrane it is defined as intraepithelial carcinoma, while if the malignant cells infiltrate only to either the lamina propria or the muscularis mucosae, it is defined as intramucosal carcinoma. There are two major clinical classification systems used for colon cancer coding. One is the tumor, node, metastasis (TNM) staging system, which was created by the American Joint Committee on Cancer (AJCC), and the other is the WHO classification system. ${ }^{6-8}$ Currently, the TNM system of the AJCC defines T1 as colon cancer that infiltrates to the submucosa, penetrating through the muscularis mucosae. Intraepithelial carcinoma and cancer with invasion only to the lamina propria are considered carcinoma in situ and de- fined as Tis. Considering the fact that colon cancer rarely metastasizes to lymph nodes due to the lack of lymphatics, the WHO classification system does not categorize intramucosal carcinoma separately and terms it as carcinoma in situ.

ICD-Oncology (ICD-O) is a classification system commonly preferred by pathologists that was created by the International Agency for Research on Cancer, an affiliate of the WHO, to register cancer. ${ }^{6}$ It is used by the National Cancer Registration of Korea, and the KCD also uses ICD-O-3 for the classification of neoplasms. According to the revised ICD$\mathrm{O}-3$, there are no changes in ICD classification compared to the previous version, except for the fact that biologic behavior codes have been added as the last digit (Table 1).

\section{PROBLEMS WITH CODING FOR EPITHELIAL NEOPLASM IN COLON}

The most important topic is which KCD code should be assigned to non-invasive high-grade neoplasms, Category 4 according to the Vienna classification. This controversy is related to the recent revision of the Vienna classification, which is the result of an agreement between pathologists of the West and Japan. Unified criteria were proposed in 1998, after a thorough discussion for gastric and colonic cancer. In particular, neoplasms with no differences in treatment were grouped together. Category 4 included non-invasive high-grade neoplasm, and intramucosal carcinoma was initially categorized as category 5.1, invasive cancer. Later, in 2000, intramucosal carcinoma was included into category 4 as 4.4, since it was difficult to differentiate whether the lamina propria was invaded, and invasion status did not affect the prognosis. However, clinicians were easily confused because stomach cancer, which uses a similar classification system, defined intramucosal carcinoma as category 5 , invasive cancer. ${ }^{3,9}$

As international guidelines for colon cancer changed, there were conferences held in Korea to discuss these matters. The Korean Society of Pathologists hosted two symposiums to

Table 1. Biologic Behavior Codes of International Classification of Diseases-Oncology-3

\begin{tabular}{cl}
\hline Code & \multicolumn{1}{c}{ Disease } \\
\hline$/ 0$ & Benign \\
$/ 1$ & Uncertain whether benign or malignant (borderline \\
& malignancy, low malignant potential, uncertain \\
& malignant potential) \\
/2 & Carcinoma in situ (intraepithelial, noninfiltrating, \\
& noninvasive) \\
/3 & Malignant, primary site \\
/6 & Malignant, metastatic site or secondary site \\
/9 & Malignant, uncertain primary or secondary site \\
\hline
\end{tabular}


discuss and conduct surveys to suggest a guideline for colon cancer classification and the ICD-O. To decrease the differences in point of view caused by different classification systems between the East and the West, high-grade dysplasia and intramucosal carcinoma were described by applying the TNM staging system of the AJCC, classifying carcinoma in situ and intramucosal tumor as pTis. The ICD-O suggested classifying high-grade dysplasia, carcinoma in situ, and intramucosal carcinoma with the biologic behavior code " $/ 2$." In a survey conducted by symposium attendees, $81.7 \%$ classified intramucosal tumor as biologic behavior code " $/ 2$," but $16.5 \%$ classified it as "/3." These results caused a public misunderstanding that it is difficult to apply a standardized classification system to cancer. In addition, clinicians were confused regarding discrepancies in opinion among the pathologists. Although there was a proposal to create an updated guideline for cancer registration of gastrointestinal tumors by the Gastrointestinal Pathology Study Group of the Korean Society of Pathologists in 2012, the pathologists' suggested describing everything observed in endoscopy with the ICD-O code of "/2" for epithelial tumors, ${ }^{5}$ and there was insufficient communication or educational support between clinicians and pathologists who were responsible for coding and medical certificates.

\section{OPINIONS OF FIRST LINE GASTROENTEROLOGISTS}

The Korean Society of Gastrointestinal Endoscopy opened a debate forum. Gastroenterologists, pathologists, lawyers, and other specialists in many fields were invited to discuss and offer solutions to the problems that had arisen with coding for colon cancer, with the goal of eventually decreasing the confusion of doctors in practice. An Internet-based survey regarding the coding of colonic epithelial tumor and subepithelial tumor was carried out beforehand and a total of 561 people (male 473, female 88) responded. In response to the question asking which code to assign to a mass that was resected endoscopically with "no lymphatic or vascular invasion, and had clean margins but invaded through the muscularis mucosae," most of the respondents (66\%) decided to classify it as C and 33\% classified it as D. Problems addressing the confusion with coding considering the depth of invasion were also discussed though this survey.

\section{MODULATION OF VIEWPOINTS OF GASTROENTEROLOGISTS AND PATHOLOGISTS}

Most of the gastroenterologists agreed to most of the concepts of the pathologists, but a few matters were not agreed upon. The conclusion was reached that coding should be done purely based on the characteristics of the disease, excluding social issues such as insurance. The idea to classify low grade adenoma, which constitutes the highest percentage, as D12, category $/ 0$, and high grade dysplasia, carcinoma in situ, intraepithelial carcinoma, and intramucosal tumor as D01, category 12 , and to classify cancer which had invaded to the submucosa as C18, C19, C20 category /3, was mostly accepted. Although this guideline may not satisfy all clinicians, it was encouraging that it shared its context with the revised Vienna classification, the WHO classification, and the AJCC staging system, as well as with the Korean pathologists (Table 2).

\section{CODING FOR NEUROENDOCRINE TUMOR OF COLON}

Recently, classification systems for neuroendocrine tumor are being revised to take into account newly discovered facts. However, in Korea, the ICD-10 and KCD include neuroendocrine tumor within the category of epithelial tumor and do not differentiate neuroendocrine tumor by its grade, which is the basis of diagnosis. ${ }^{10}$

Neuroendocrine malignancy shows different clinical features according to its origin, histological differentiation, secreted hormones, and biological factors. However, organizations involved in classification such as the WHO, European Neuroendocrine Tumor Society (ENETS), and AJCC define neuroendocrine tumor differently, intensifying the confusion. ${ }^{11-13}$ In the WHO classification established in 2000, neuroendocrine tumor is divided by histological differentiation and clinical features into well-differentiated endocrine carcinoid, well-differentiated neuroendocrine carcinoma, and poorly differentiated neuroendocrine carcinoma. The classification was later revised in 2010, with the tumor divided by its mitotic count and Ki67 index into G1, G2, and G3. G1 and G2 are classified as neuroendocrine tumor and G3 is classified as neuroendocrine carcinoma (Table 3). ${ }^{13}$ However, any neuroendocrine tumor consisting of malignant cells is classified as neuroendocrine carcinoma irrespective of the mitotic count and Ki67 index. Neuroendocrine tumor may also be classified

Table 2. Proposal of Codes for Colonic Epithelial Tumors

\begin{tabular}{lc}
\hline \multicolumn{1}{c}{ Results } & Code \\
\hline Adenoma, low grade dysplasia & D12 \\
Hyperplastic polyp & K635 \\
Adenoma, high grade dysplasia & D01 \\
Carcinoma in situ & D01 \\
Intraepithelial carcinoma & D01 \\
Intramucosal carcinoma & D01 \\
\hline
\end{tabular}


Table 3. Grading System for Gastroenteropancreatic Neuroendocrine Tumors ${ }^{7}$

\begin{tabular}{ccc}
\hline Grade & Mitotic count, 10 HPFs & Ki-67 index, \% \\
\hline G1 & 1 & $\leq 2$ \\
G2 & $2-20$ & $3-20$ \\
G3 & $>20$ & $>20$ \\
\hline
\end{tabular}

HPF, high power field.

according to its origin. Enterochromaffin cell neuroendocrine tumors and serotonin-producing neuroendocrine tumors originate from the mid-gut, while glucagon-like peptide-producing and pancreatic polypeptide/polypeptide YY (PP/ PYY)-producing neuroendocrine tumors originate from the L-cells of the hindgut. Eighty percent of the neuroendocrine tumors in the rectum express markers of L-cells. The 2010 WHO classification categorized L-cell type neuroendocrine tumors as $/ 1$ and other neuroendocrine tumors as $/ 3$. However, since specific diagnostic criteria or special stains for L-cells were not suggested and methods to distinguish glucagon-like peptide and PP/PYY were not established, additional studies are being conducted.

In clinical practice, neuroendocrine tumor of the rectum is usually found incidentally, tends to be smaller than $1 \mathrm{~cm}$ and well-differentiated, and is limited to the mucosa and submucosa. ${ }^{14,15}$ As a result, the possibility of metastasis is low, with patients showing a greater than $99 \% 10$-year survival rate. If the tumor invades though the muscularis or is larger than 2 $\mathrm{cm}$ in size, the possibility of metastasis increases. In the WHO classification, it is defined as "benign" if the tumor is under 1 $\mathrm{cm}$ in size, limited to the mucosa or submucosa, and does not have vascular invasion. However, in the 2010 WHO classification, the L-cell type is classified as / 1 without any mention of the size. However, only a few neuroendocrine tumors in the rectum measuring less than $1 \mathrm{~cm}$ have been reported to metastasize to the liver. Therefore, it is more suitable to classify them as / 1 rather than benign, as mentioned in the 2000 WHO guideline.

In conclusion, revisions are needed with a greater amount of accumulated data, since no other standards such as size, grade, or vascular invasion are established except for the fact that the L-cell type is classified as / 1 , while accurate diagnostic methods or standards need to be established for the L-cell type. ${ }^{5}$

\section{QUESTIONNAIRE FOR GASTROENTEROLOGISTS ON NEUROENDOCRINE TUMOR}

An Internet-based survey based on the subepithelial tumor was conducted, focused on a specimen obtained by endoscop-
Table 4. Proposal of Codes for Colonic Neuroendocrine Tumors

\begin{tabular}{lc}
\hline \multicolumn{1}{c}{ Results } & Code \\
\hline Neuroendocrine tumor, colon & C18 \\
Rectal neuroendocrine tumor, grade 1 & \\
Lymph node/distant metastasis & C20 \\
Muscle/angioinvasion & C20 \\
$>2 \mathrm{~cm}$ & $\mathrm{C} 20$ \\
$1-2 \mathrm{~cm}$ & $\mathrm{C} 20$ \\
$<1 \mathrm{~cm}$ & $\mathrm{D} 37$ \\
\hline
\end{tabular}

ic submucosal dissection. It was a grade 1 neuroendocrine tumor measuring $1.5 \mathrm{~cm}$, limited to the submucosa, with no lymphovascular invasion and clean resection margins. The Ki67 labeling index was less than $1 \%$ and the mitotic count was 0/6 high power fields (HPFs). Among the respondents, 164 (29.2\%) classified it as C, 47 (8.4\%) coded it D01, 298 (53.1\%) coded it D37, and $50(8.9 \%)$ coded it D12; these responses were diverse and represent the confusion in clinical practice. The criteria used by the respondents to classify the tumor as malignant were also diverse. One hundred forty-six respondents (26\%) stated that they would assign a C code if the tumor was carcinoid, $26(4.6 \%)$ would assign a $\mathrm{C}$ code if the tumor was over $6 \mathrm{~mm}$ in size, 252 (44.9\%) would assign a C code if the tumor was over $10 \mathrm{~mm}$ in size, and $96(17.1 \%)$ would assign a $\mathrm{C}$ code if the tumor was over $20 \mathrm{~mm}$ in size.

\section{AGREEMENT WITH CODING OF NEUROENDOCRINE TUMOR}

The necessity to proceed with caution concerning with the coding of neuroendocrine tumor was highlighted. According to the scientific grounds, the decisions should be based on the location, origin, differentiation, and size, since these are the key factors in prognosis.

Colonic subepithelial neuroendocrine tumor, especially that located in the rectum, should be given the biological behavior code " $/ 1$ " if it is smaller than $1 \mathrm{~cm}$ in size, ENETS grade 1, limited to the mucosa and submucosa, and does not show any angioinvasion. ${ }^{5}$ Since some of these cases are rarely reported to show metastasis, it may not be sufficient to classify it as a benign neoplasm as in the $2000 \mathrm{WHO}$ guideline, but rather code it as D37, neoplasm of uncertain or unknown behavior of the digestive system. If any of these conditions do not fit, it should be classified as "/3" with a C code (Table 4).

\section{CONCLUSIONS}

The background and the problems associated with the coding system for colonic epithelial tumor and subepithelial tu- 
mor were discussed in this article. We expect that an appropriate classification system be developed based on social agreement following constructive discussions in the future.

\section{Conflicts of Interest}

The authors have no financial conflicts of interest.

\section{REFERENCES}

1. Kim HG, Kim JO, Lee SH, et al. Clinical classification of colorectal epithelial tumors and proposal for diagnostic coding. Intest Res 2011;9:1-11.

2. World Health Organization; WHO Collaborating Centres for Classification of Diseases. International Statistical Classification of Diseases and Related Health Problems, Tenth Revision. 2nd ed. Geneva: World Health Organization; 2004.

3. Participants in the Paris Workshop. The Paris endoscopic classification of superficial neoplastic lesions: esophagus, stomach, and colon: November 30 to December 1, 2002. Gastrointest Endosc 2003;58(6 Suppl):S3-S43.

4. Cho MY, Kang YK, Kim KM, et al. Porposal for creating a guideline for cancer registration of the gastrointestinal tumors (I). Korean J Pathol 2008;42:140-150.

5. Jung ES, Kang YK, Cho MY, et al. Update on the proposal for creating a guideline for cancer registration of the gastrointestinal tumors (I-2). Korean J Pathol 2012;46:443-453.
6. Fritz A, Percy C, Jack A, et al. International Classification of Diseases for Oncology: ICD-O. 3rd ed. Geneva: World Health Organization; 2000.

7. Edge SB, Byrd DR, Compton CC, Fritz AG, Greene FL, Trotti A. AJCC Cancer Staging Manual. 7th ed. New York: Springer-Verlag; 2010.

8. Bosman FT, Carneiro F, Hruban RH, Theise ND. WHO Classification of Tumours of the Digestive System. 4th ed. Lyon: IARC Press; 2010.

9. Schlemper RJ, Riddell RH, Kato Y, et al. The Vienna classification of gastrointestinal epithelial neoplasia. Gut 2000;47:251-255.

10. Kim BC, Park CH, Kim TI, et al. Variable clinical classifications and diagnostic coding systems of colorectal neuroendocrine tumor. Intest Res 2013;11:14-22.

11. Rindi G, Klöppel G, Couvelard A, et al. TNM staging of midgut and hindgut (neuro) endocrine tumors: a consensus proposal including a grading system. Virchows Arch 2007;451:757-762.

12. Rindi G, Bordi C, Rappel S, La Rosa S, Stolte M, Solcia E. Gastric carcinoids and neuroendocrine carcinomas: pathogenesis, pathology, and behavior. World J Surg 1996;20:168-172.

13. Ramage JK, Goretzki PE, Manfredi R, et al. Consensus guidelines for the management of patients with digestive neuroendocrine tumours: well-differentiated colon and rectum tumour/carcinoma. Neuroendocrinology 2008;87:31-39.

14. Park $\mathrm{CH}$, Cheon JH, Kim JO, et al. Criteria for decision making after endoscopic resection of well-differentiated rectal carcinoids with regard to potential lymphatic spread. Endoscopy 2011;43:790-795.

15. Soga J. Early-stage carcinoids of the gastrointestinal tract: an analysis of 1914 reported cases. Cancer 2005;103:1587-1595. 